\title{
Conservatism of Passivity Criteria for Stability Analysis of Trilateral Haptic Systems
}

\author{
Jian Li ${ }^{*} \quad$ Mahdi Tavakoli ${ }^{\dagger} \quad$ Victor Mendez ${ }^{\star} \quad$ Qi Huang ${ }^{\S}$ \\ * School of Energy Science and Engineering, University of Electronic Science and Technology of China, \\ Chengdu, Sichuan, China 611731 \\ $* \dagger$ Department of Electrical and Computer Engineering, University of Alberta, Edmonton, AB T6G 2V4, Canada
}

\begin{abstract}
Trilateral haptic systems can be modeled as three-port networks. Analysis of coupled stability of a three-port network can be accomplished in either the passivity or the absolute stability frameworks assuming all three ports are connected to passive but otherwise unknown terminations. This paper first reviews our recent results in terms of passivity and absolute stability criteria for general threeport networks - both criteria are founded on the properties of a positive-real Hermitian matrix. Next, we show that the absolute stability criterion is less conservative than the passivity criterion and that the two criteria become the same when the trilateral system is represented by a reciprocal immitance matrix. Then, to show how the two criteria may be utilized at the system design stage, we apply them to the problem of designing controllers for a dual-user haptic teleoperation system. Using the two criteria, controllers are then designed and compared in terms of conservatism and performance in simulations.
\end{abstract}

Index Terms: H.5.2 [Information Interfaces and Presentation]: User Interfaces-Haptic I/O; I.2.9 [Artificial Intelligence]: Problem Solving, Control Methods, and Search-Control theory

\section{INTRODUCTION}

New application of multilateral teleoperation systems have recently emerged including collaboration of multiple users to perform a haptic virtual task and shared control of a robot in a remote environment by multiple users. Practical uses of these include telerehabilitation, surgical training, and cooperative multi-robot systems. An interesting class of multilateral haptic systems is the trilateral one, which can be modeled as a three-port network.

In designing haptic teleoperation controllers, the main goals are performance and stability. For a bilateral teleoperation system consisting of a teleoperator (master, slave and controllers) coupled to terminations (human operator and environment), performance is the ability of a teleoperation system to present the undistorted dynamics of the environment to the human operator. This requires that the master and the slave positions and interactions match regardless of the operator and environment dynamics. Taking precedence to performance is stability, which is necessary for safe teleoperation. Investigation of teleoperation system stability requires not only the teleoperator's immitance $(z, y, h, g)$ parameters, but also the models of the human operator and the environment, which are usually unknown, uncertain, and/or time-varying. Consequently, conventional techniques cannot be used to study the stability of teleoperation systems. Methods for analyzing the stability of teleoperation

\footnotetext{
*e-mail: jian1@ualberta.ca

†e-mail:mahdi.tavakoli@ualberta.ca

†e-mail: vmendez@ualberta.ca

$\S$ e-mail:huangqi@uestc.edu.cn
}

IEEE World Haptics Conference 2013

14-18 April, Daejeon, Korea

978-1-4799-0088-6/13/\$31.00 @2013 IEEE system can be categorized as teleoperator passivity and teleoperator absolute stability criteria. By definition, a teleoperator is passive if the total energy delivered to it by its terminations is non-negative. Also, by definition, an teleoperator is absolutely stable if the teleoperation system remains stable for all possible passive terminations. For bilateral teleoperation systems comprising one master and one slave, teleoperator passivity and absolute stability can be analyzed via Raisbeck's criterion [1] and Llewellyn's criterion [2], respectively. In this paper, we discussed their counterparts for a trilateral teleoperation system.

For absolute stability analysis of a trilateral teleoperator, in [3], and [4] the authors proposed methods in which the three-port network model of the teleoperator is reduced to a two-port network by assuming a known termination for the third port, paving the way for the application of Llewellyn's criterion. Unfortunately, in the above approaches a degree of freedom is lost when the third port is coupled to a known termination. In [5], the stability of the nonreciprocal $n$-port network was studied by finding a reciprocal $n$-port network with the same stability characteristics as the nonreciprocal $n$-port network in question. For the reciprocal $n$-port network, absolute stability can be studied through its equivalence to passivity. This method can be lengthy for the general $n$-port networks; however, the method is tractable for the case of $n=3$ (three-port networks).

For passivity analysis of a trilateral teleoperator, Wang et al. [6] proposed three different passive four-channel architectures. The passive control architectures can ensure stability even in the case of large time delays. Shahbazi et al. [7] performed stability analysis for dual-user teleoperation systems (three-port networks) by using the passivity theory. In [8], Mendez et al. presented a criterion for passivity of $n$-port networks with unknown terminations. The criterion gives the necessary and sufficient conditions for passivity of the $n$-port network assuming that the unknown terminations (human operators and environments) are passive.

In this paper, a comparison on the performance between our newly-developed absolute stability criterion and Mendez's [8] passivity criterion for three-port networks is provided. In a case study involving a dual-user haptic system, each of these two criteria is used for the design of stabilizing controllers and the results are compared in terms of conservatism and performance. The rest of the paper is organized as follows: The next section gives mathematical definitions and lemmas for analysis of the passivity and absolute stability. In Section 3, the proposed passivity and absolute stability criteria for three-port networks are derived. We show the conservatism of the passivity conditions on compared to the absolute stability conditions. Then, in Section 4, the passivity and absolute stability criterion are used in designing a trilateral shared control architecture for a dual-user teleoperation system. The passivity and absolute stability conditions in terms of system parameters including controller gains are found. Finally, simulations to verify the validity of the calculated passivity and absolute stability conditions for a position-position dual-user teleoperation system and a comparison of the performance between these two methods are 


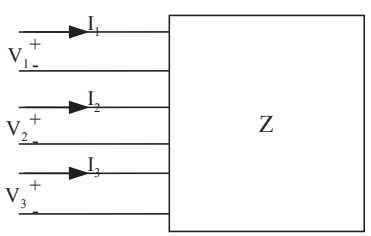

(a)

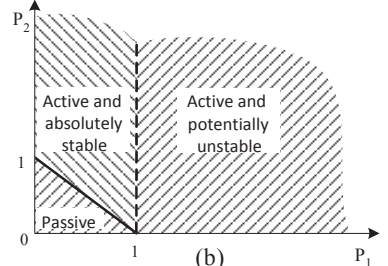

(b)
Figure 1: (a) Three-port network, (b) Stability-activity diagram.

presented in Section 5. Section 6 contains concluding remarks.

\section{Mathematical Preliminaries}

Definition 1. [9] A Hermitian matrix is a complex square matrix that is equal to its conjugate transpose.

Property 1. [10] If $M$ is a Hermitian matrix, it is positive definite (positive semidefinite) if its leading principal minors are all positive (nonnegative).

Lemma 1. [11] A linear time-invariant minimal realization model with transfer matrix $G(s)$ is passive (strictly passive) if $G(s)$ is positive real (strictly positive real).

Lemma 2. [11] A $n \times n$ proper rational transfer matrix $G(s)$ is called positive real if

i) Poles of all elements of $G(s)$ are in $\operatorname{Re}[s] \leq 0$,

ii) Any pure imaginary pole $j \omega$ of any element of $G(s)$ is a simple pole and the residue matrix $\lim _{s \rightarrow j \omega}(s-j \omega) G(s)$ is positive semidefinite Hermitian,

iii) For all real $\omega$ for which $j \omega$ is not a pole of any element of $G(s)$, the matrix $G(j \omega)+G^{T}(-j \omega)$ is positive semidefinite.

\section{Passivity and Absolute Stability of Three-Port NETWORKS}

For two-port networks, two well-known methods - Llewellyn's absolute stability criterion [2] and Raisbeck's passivity criterion [1] have been developed to investigate the stability of the network when connected to arbitrary passive terminations. As shown by Haykin [12], these criteria are equivalent if and only if the two-port network is symmetric. In the general case, all passive two-port network are absolutely stable but not vice versa.

In this paper, we will discuss conditions for the passivity and absolute stability of three-port networks. We will show that these criteria are equivalent if and only if the three-port network is symmetric, and compare the two in the general cases in terms of conservativeness.

Consider a general nonreciprocal three-port network, as shown in Figure 1-(a), with the impedance matrix

$$
Z=\left[\begin{array}{lll}
Z_{11} & Z_{12} & Z_{13} \\
Z_{21} & Z_{22} & Z_{23} \\
Z_{31} & Z_{32} & Z_{33}
\end{array}\right]
$$

Let $Z_{m n}=r_{m n}+j x_{m n}, m, n=1,2,3$. We have the following theorems for the passivity and absolute stability of the three-port network modeled by $Z$.

\subsection{Passivity theorem}

Theorem 1. A three-port network with the impedance matrix $Z$ in (1) is passive if and only if

1. The $Z$ matrix elements have no poles in the right-half plane (RHP).

2. Any poles of the $Z$ matrix elements on the imaginary axis are simple, and the residues of the $Z$ matrix elements at these poles satisfy the following conditions:

$$
\begin{aligned}
& k_{m m} \geq 0, \quad m=1,2,3 \\
& \frac{k_{11} k_{22}-k_{12} k_{21}}{k_{11}} \geq 0 \\
& \frac{k_{11} k_{33}-k_{13} k_{31}}{k_{11}}-\frac{k_{11} k_{23}-k_{21} k_{13}}{k_{11} k_{22}-k_{12} k_{21}} \frac{k_{11} k_{32}-k_{31} k_{12}}{k_{11}} \geq 0
\end{aligned}
$$

where $k_{m n}$ denotes the residue of $Z_{m n}$.

3. The real and imaginary part of the $Z$ matrix elements satisfy the following inequalities

$$
\begin{aligned}
& r_{11} \geq 0 \\
& r_{22} \geq 0 \\
& r_{33} \geq 0 \\
& 4 r_{11} r_{22}-\left(r_{12}+r_{21}\right)^{2}-\left(x_{12}-x_{21}\right)^{2} \geq 0 \\
& 4 r_{11} r_{22} r_{33}-r_{33}\left[\left(r_{12}+r_{21}\right)^{2}+\left(x_{12}-x_{21}\right)^{2}\right] \\
& -r_{22}\left[\left(r_{13}+r_{31}\right)^{2}+\left(x_{13}-x_{31}\right)^{2}\right] \\
& -r_{11}\left[\left(r_{23}+r_{32}\right)^{2}+\left(x_{23}-x_{32}\right)^{2}\right] \\
& +\left(r_{23}+r_{32}\right)\left(r_{13}+r_{31}\right)\left(r_{12}+r_{21}\right) \\
& +\left(r_{12}+r_{21}\right)\left(x_{13}-x_{31}\right)\left(x_{23}-x_{32}\right) \\
& -\left(r_{13}+r_{31}\right)\left(x_{12}-x_{21}\right)\left(x_{23}-x_{32}\right) \\
& +\left(r_{23}+r_{32}\right)\left(x_{13}-x_{31}\right)\left(x_{12}-x_{21}\right) \geq 0
\end{aligned}
$$

Proof. According to Lemma 1, the three-port network is passive if and only if its transfer matrix (i.e., the matrix $Z$ in (1)) is positive real, which can be verified through Lemma 2 . It is obvious that Condition 1 in the theorem is the same as Condition (i) in Lemma 2. Let

$$
K=\left[\begin{array}{lll}
k_{11} & k_{12} & k_{13} \\
k_{21} & k_{22} & k_{23} \\
k_{31} & k_{32} & k_{33}
\end{array}\right]
$$

denote the residue matrix of the $Z$ matrix elements. According to Condition (ii) in Lemma 2, $K$ must be positive semidefinite Hermitian. Based on Property $1, K$ is positive semidefinite if its leading principal minors are all nonnegative, i.e.,

$$
\begin{aligned}
& k_{m m} \geq 0, \quad m=1,2,3 \\
& k_{11} k_{22}-k_{12} k_{21} \geq 0 \\
& k_{11} k_{22} k_{33}-k_{11} k_{23} k_{32}-k_{22} k_{13} k_{31}-k_{33} k_{12} k_{21} \\
& +k_{12} k_{23} k_{31}-k_{13} k_{21} k_{32} \geq 0
\end{aligned}
$$

The above inequalities are equivalent to those in Condition 2 of Theorem 1.

According to Condition (iii) of Lemma 2, the Hermitian matrix

$$
Z(j \omega)+Z^{T}(-j \omega)=\left[\begin{array}{ccc}
2 r_{11} & r_{12}+r_{21} & r_{13}+r_{31} \\
r_{12}+r_{21} & 2 r_{22} & r_{23}+r_{32} \\
r_{13}+r_{31} & r_{23}+r_{32} & 2 r_{33}
\end{array}\right]
$$




$$
+j\left[\begin{array}{ccc}
0 & x_{12}-x_{21} & x_{13}-x_{31} \\
x_{21}-x_{12} & 0 & x_{23}-x_{32} \\
x_{31}-x_{13} & x_{32}-x_{23} & 0
\end{array}\right]
$$

needs to be positive semidefinite. Using Property 1 , this leads us to Conditions (2a)-(2e).

\subsection{Absolute stability theorem}

Theorem 2. A three-port network with impedance matrix $Z$ in (1) satisfying the symmetrization condition

$$
Z_{13} Z_{21} Z_{32}-Z_{12} Z_{23} Z_{31}=0
$$

is absolutely stable if and only if Conditions 1 and 2 in Theorem 1 hold and, for all real values of frequencies $\omega$, we have

$r_{11} \geq 0$

$r_{22} \geq 0$

$r_{33} \geq 0$

$r_{11} r_{22}-\frac{\left|Z_{12} Z_{21}\right|+\operatorname{Re}\left(Z_{12} Z_{21}\right)}{2} \geq 0$

$r_{11} r_{22} r_{33}-r_{11} \frac{\left|Z_{23} Z_{32}\right|+\operatorname{Re}\left(Z_{23} Z_{32}\right)}{2}$

$-r_{22} \frac{\left|Z_{13} Z_{31}\right|+\operatorname{Re}\left(Z_{13} Z_{31}\right)}{2}$

$-r_{33} \frac{\left|Z_{12} Z_{21}\right|+\operatorname{Re}\left(Z_{12} Z_{21}\right)}{2}$

$+2 \operatorname{Re}\left(\sqrt{Z_{12} Z_{21}}\right) \operatorname{Re}\left(\sqrt{Z_{13} Z_{31}}\right) \operatorname{Re}\left(\sqrt{Z_{23} Z_{32}}\right) \geq 0$

Proof. [13] If and only if the symmetrization condition (8) holds, the general asymmetric matrix $Z$ in (1) has the same stability characteristics as the following equivalent symmetric matrix:

$$
Z_{e q}=\left[\begin{array}{ccc}
Z_{11} & \gamma_{1} \sqrt{Z_{12} Z_{21}} & \gamma_{2} \sqrt{Z_{13} Z_{31}} \\
\gamma_{1} \sqrt{Z_{12} Z_{21}} & Z_{22} & \gamma_{3} \sqrt{Z_{23} Z_{32}} \\
\gamma_{2} \sqrt{Z_{13} Z_{31}} & \gamma_{3} \sqrt{Z_{23} Z_{32}} & Z_{33}
\end{array}\right]
$$

where $\gamma_{i}= \pm 1$ for $i=1,2,3$. This happens because $Z$ and $Z_{e q}$ have identical principal minors of all orders and therefore, according to [14], we get $\operatorname{det}\left(Z+Z_{0}\right)=\operatorname{det}\left(Z_{e q}+Z_{0}\right)$ for all passive $Z_{0}=\operatorname{diag}\left[z_{1}, z_{2}, z_{3}\right]$.

Now, the three-port network with symmetric transfer matrix $Z_{e q}$ is absolutely stable if and only if it is passive [15]. According to Lemma $1, Z_{e q}$ is passive if and only if it is nonnegative real, which can be verified through Lemma 2. Thus, it is found that Conditions 1 and 2 in Theorem 1 need to hold. Additionally, according to Condition (iii) of Lemma 2, the Hermitian matrix

$$
\begin{gathered}
Z_{e q}(j \omega)+Z_{e q}^{T}(-j \omega)= \\
{\left[\begin{array}{ccc}
2 r_{11} & 2 \gamma_{1} \operatorname{Re} \sqrt{Z_{12} Z_{21}} & 2 \gamma_{2} \operatorname{Re} \sqrt{Z_{13} Z_{31}} \\
2 \gamma_{1} \operatorname{Re} \sqrt{Z_{12} Z_{21}} & 2 r_{22} & 2 \gamma_{3} \operatorname{Re} \sqrt{Z_{23} Z_{32}} \\
2 \gamma_{2} \operatorname{Re} \sqrt{Z_{13} Z_{31}} & 2 \gamma_{3} \operatorname{Re} \sqrt{Z_{23} Z_{32}} & 2 r_{33}
\end{array}\right]}
\end{gathered}
$$

needs to be positive semidefinite. Using Property 1 and

$$
\left(\operatorname{Re}\left(\sqrt{Z_{m n} Z_{n m}}\right)\right)=\sqrt{\frac{\left|Z_{m n} Z_{n m}\right|+\operatorname{Re}\left(Z_{m n} Z_{n m}\right)}{2}}
$$

where $m, n=1,2,3$ lead us to Conditions (9a)-(9e).

Remark 1. Theorem 1 and Theorem 2 hold not only for the impedance matrix (1) of a general network but also for its other immitance matrices $(y, h, g)$. The reason for this is that a gyration operator, which can transform on immitance matrix to another, preserves passivity [16].

\subsection{Comparison of passivity and absolute stability the- orem}

Conditions 1 and 2 of the passivity theorem are in common with the absolute stability theorem, and any difference lies in Conditions 3. Also, as part of Condition 3 in Theorem 1, (2a)-(2c) in the passivity theorem are the same as (9a)-(9c) in the absolute stability theorem. Now, Condition (9d) for absolute stability can be re-written as

$$
\frac{\left(\operatorname{Re}\left(\sqrt{Z_{12} Z_{21}}\right)\right)^{2}}{r_{11} r_{22}} \leq 1
$$

while Condition (2d) for passivity can be manipulated into the form

$$
\frac{\left(\operatorname{Re}\left(\sqrt{Z_{12} Z_{21}}\right)\right)^{2}}{r_{11} r_{22}}+\frac{\left(\left|Z_{12}\right|-\left|Z_{21}\right|\right)^{2}}{4 r_{11} r_{22}} \leq 1
$$

Obviously, the passivity condition (14) or the equivalent (2d) is more conservative than the absolute stability condition (13) or the equivalent (9d). These two conditions are equivalent if and only if $Z_{12}=Z_{21}$.

Furthermore, Condition (9e) for absolute stability can be rewritten as

$$
\begin{aligned}
& P_{1}= \\
& \frac{\left(\operatorname{Re}\left(\sqrt{Z_{23} Z_{32}}\right)\right)^{2}}{r_{22} r_{33}}+\frac{\left(\operatorname{Re}\left(\sqrt{Z_{13} Z_{31}}\right)\right)^{2}}{r_{11} r_{33}}+\frac{\left(\operatorname{Re}\left(\sqrt{Z_{12} Z_{21}}\right)\right)^{2}}{r_{11} r_{22}} \\
& -\frac{2 \operatorname{Re}\left(\sqrt{Z_{12} Z_{21}}\right) \operatorname{Re}\left(\sqrt{Z_{13} Z_{31}}\right) \operatorname{Re}\left(\sqrt{Z_{23} Z_{32}}\right)}{r_{11} r_{22} r_{33}} \leq 1
\end{aligned}
$$

On the other hand, noting that

$$
\begin{aligned}
& \left(r_{m n}+r_{n m}\right)^{2}+\left(x_{m n}-x_{n m}\right)^{2} \\
& =4\left(\operatorname{Re}\left(\sqrt{Z_{m n} Z_{n m}}\right)\right)^{2}+\left(\left|Z_{m n}\right|-\left|Z_{n m}\right|\right)^{2}
\end{aligned}
$$

where $m, n=1,2,3$, Condition (2e) for passivity can be manipulated into the form

$$
P_{1}+P_{2} \leq 1
$$

In the following, we will show that $P_{2} \geq 0$, establishing the fact that the passivity condition (17) or the equivalent (2e) is more conservative than the absolute stability condition (15) or the equivalent $(9 \mathrm{e})$. These two are equivalent if and only if $Z_{12}=Z_{21}, Z_{13}=Z_{31}$, and $Z_{23}=Z_{32}$.

In (17), we have $P_{2}=Q_{1}+Q_{2}-Q_{3}$ where

$$
\begin{aligned}
& Q_{1}=\frac{r_{11}\left(\left|Z_{23}\right|-\left|Z_{32}\right|\right)^{2}}{4 r_{11} r_{22} r_{33}}+\frac{r_{22}\left(\left|Z_{13}\right|-\left|Z_{31}\right|\right)^{2}}{4 r_{11} r_{22} r_{33}} \\
& +\frac{r_{33}\left(\left|Z_{12}\right|-\left|Z_{21}\right|\right)^{2}}{4 r_{11} r_{22} r_{33}} \\
& Q_{2}=\frac{2 \operatorname{Re} \sqrt{Z_{12} Z_{21}} \operatorname{Re} \sqrt{Z_{13} Z_{31}} \operatorname{Re} \sqrt{Z_{23} Z_{32}}}{r_{11} r_{22} r_{33}} \\
& Q_{3}=\frac{\left(r_{12}+r_{21}\right)\left(r_{13}+r_{31}\right)\left(r_{23}+r_{32}\right)}{4 r_{11} r_{22} r_{33}} \\
& +\frac{\left(r_{12}+r_{21}\right)\left(x_{13}-x_{31}\right)\left(x_{23}-x_{32}\right)}{4 r_{11} r_{22} r_{33}} \\
& -\frac{\left(r_{13}+r_{31}\right)\left(x_{12}-x_{21}\right)\left(x_{23}-x_{32}\right)}{4 r_{11} r_{22} r_{33}} \\
& +\frac{\left(r_{23}+r_{32}\right)\left(x_{13}-x_{31}\right)\left(x_{12}-x_{21}\right)}{4 r_{11} r_{22} r_{33}}
\end{aligned}
$$

Obviously, $Q_{1} \geq 0$. Because of (12), $Q_{2} \geq 0$. Therefore, if $Q_{3}<0$, then $P_{2}>0$. When $Q_{3}>0$, then $P_{2} \geq 0$ if and only if $\left(Q_{1}+Q_{2}\right)^{2}-$ $Q_{3}^{2} \geq 0$, which amounts to 


$$
\begin{aligned}
& \left(\left|Z_{12}\right|-\left|Z_{21}\right|\right)^{2}\left[16 r_{33} r_{123}+4 r_{11} r_{33}\left(\left|Z_{23}\right|-\left|Z_{32}\right|\right)^{2}\right. \\
& \left.-4\left(\operatorname{Re} \sqrt{Z_{13} Z_{31}}\right)^{2}\left(\left(r_{23}+r_{32}\right)^{2}+\left(x_{23}-x_{32}\right)^{2}\right)\right] \\
& +\left(\left|Z_{13}\right|-\left|Z_{31}\right|\right)^{2}\left[16 r_{22} r_{123}+4 r_{22} r_{33}\left(\left|Z_{12}\right|-\left|Z_{21}\right|\right)^{2}\right. \\
& \left.-4\left(\operatorname{Re} \sqrt{Z_{23} Z_{32}}\right)^{2}\left(\left(r_{12}+r_{21}\right)^{2}+\left(x_{12}-x_{21}\right)^{2}\right)\right] \\
& +\left(\left|Z_{23}\right|-\left|Z_{32}\right|\right)^{2}\left[16 r_{11} r_{123}+4 r_{11} r_{22}\left(\left|Z_{13}\right|-\left|Z_{31}\right|\right)^{2}\right. \\
& \left.-4\left(\operatorname{Re} \sqrt{Z_{12} Z_{21}}\right)^{2}\left(\left(r_{13}+r_{31}\right)^{2}+\left(x_{13}-x_{31}\right)^{2}\right)\right] \geq 0
\end{aligned}
$$

where $r_{123}=\operatorname{Re} \sqrt{Z_{12} Z_{21}} \operatorname{Re} \sqrt{Z_{13} Z_{31}} \operatorname{Re} \sqrt{Z_{23} Z_{32}}$. It is easy to show that (18) holds in general.

In the stability-activity diagram of Figure 1(b), we have graphically represented (15) and (17) in a two-dimensional space by choosing $P_{1}$ and $P_{2}$ as the two coordinates. Evidently, all passive three-port networks are absolutely stable, but not all absolutely stable three-port networks are passive.

Remark 2. The passivity criterion of three-port network in Theorem 1 is equivalent to the absolute stability criterion in Theorem 2 if and only if the impedance matrix $Z$ in (1) is symmetric, i.e.,

$$
Z_{12}=Z_{21}, \quad Z_{13}=Z_{31}, \quad Z_{23}=Z_{32} .
$$

This holds not only for the impedance parameters of a general network but also for its others immitance parameters.

Remark 3. For teleoperation control systems, using the absolute stability criterion will allow for higher transparency compared to using the passivity theorem. The reason for this is that passivity criterion is more restrictive than the absolute stability criterion. In the case studies that will follow higher teleoperation transparency under absolute stability conditions compared to passivity conditions will be shown.

\section{Case Study: application of the Comparison of TRILATERAL HAPTIC SYSTEMS}

In this section, the aim is to apply the proposed comparative between passivity and absolute stability for trilateral haptic systems. A trilateral haptic system may be a collaborative haptic virtual environment with three users, or a dual-user haptic teleoperation system with one slave robot. In the following, for brevity, we only consider the latter and a similar procedure case may be followed for the former. We begin by reviewing a four-channel, dual-user teleoperation system and specifically investigate the stability of position-position control scheme.

In a dual-user teleoperation control system, the goal is that two users collaboratively control a robot. Such a system consists of two master robots as haptic interfaces for the two users and one slave robot to perform a desired task on an environment. This finds application in many real-world scenarios such as when the aim is to train a novice trainee (user 1) to do a task under haptic guidance from a mentor (user 2). As elaborated by [17, 4], the reference position and force for each robot are sums of positions and forces of the other two robots weighted by a parameter $\alpha \in[0,1]$ that specifies their relative control authorities. Therefore, $\alpha$ affects how the trainee and the mentor collaborate and contribute to the reference position for the slave and what share of force feedback each of them receives. For instance, if $\alpha=0$, the slave robot will be completely controlled by the mentor and the trainee will receive large force feedback urging him/her to follow the mentor's motions. On the other hand, if $\alpha=1$, the slave robot is completely controlled by the trainee, allowing the mentor to assess the skill level of the trainee by feeling the reflected forces. If $0<\alpha<1$, the trainee and the mentor collaborate and each contribute to the slave robot position while receiving some force feedback.

Consider the dual-user teleoperation system shown in Figure 2. The dynamics of the two masters and the slave in contact with the

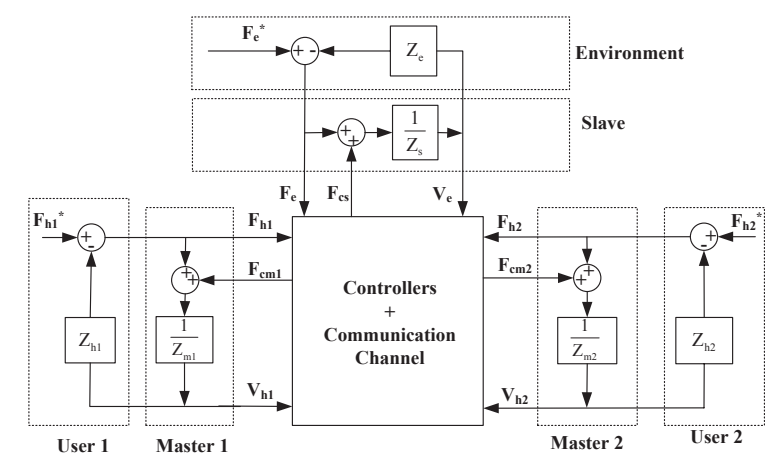

Figure 2: A dual-user haptic teleoperation system under four-channel control.

two users and the environment, respectively, are

$$
\left\{\begin{array}{l}
Z_{m i} V_{h i}=F_{h i}+F_{c m i} \\
Z_{s} V_{e}=F_{e}+F_{c s}
\end{array}\right.
$$

where $i=1,2$, and $Z_{m i}$ and $Z_{s}$ are the impedances of the two masters and the slave, respectively. Also, $F_{h i}$ denotes the interaction force between the two users and the two masters and $F_{e}$ denotes the interaction force between the slave and the environment. Lastly, $V_{h i}$, and $V_{e}$ are the users' and the environment's velocities.

The four-channel shared control laws in Figure 2 are [4, 8]:

$\left\{\begin{array}{l}F_{c m i}=-C_{m i} V_{h i}-C_{4 m i} V_{h i d}+C_{6 m i} F_{h i}-C_{2 m i} F_{h i d} \\ F_{c s}=-C_{s} V_{e}+C_{1} V_{e d}+C_{5} F_{e}+C_{3} F_{e d}\end{array}\right.$

where $C_{m i}$ and $C_{s}$ are local position controllers, $C_{6 m i}$ and $C_{5}$ are local force controllers, and $C_{1}, C_{2 m i}, C_{3}$, and $C_{4 m i}$ are feedforward and feedback compensators. Also, $V_{\text {hid }}$ and $V_{e d}$ are the reference velocities and $F_{h i d}$ and $F_{e d}$ are the references forces for the two masters and the slave, where

$\begin{array}{ll}V_{h 1 d}=\alpha V_{e}+(1-\alpha) V_{h 2}, & V_{h 2 d}=(1-\alpha) V_{e}+\alpha V_{h 1} \\ V_{e d}=\alpha V_{h 1}+(1-\alpha) V_{h 2}, & F_{h 1 d}=\alpha F_{e}+(1-\alpha) F_{h 2} \\ F_{h 2 d}=(1-\alpha) F_{e}+\alpha F_{h 1}, & F_{e d}=\alpha F_{h 1}+(1-\alpha) F_{h 2}\end{array}$

It is easy to verify that the reference velocities (positions) and references forces in (22) are consistent with the trainee/mentor collaboration scenario discussed above.

Position-position control is a special case of dual-user shared control in which there is no need for any force sensor measurements [18]. In this control architecture, we have $C_{2 m 1}=C_{2 m 2}=$ $C_{3}=C_{5}=C_{6 m 1}=C_{6 m 2}=0$. For good position tracking, the common choice is $C_{1}=C_{s}, C_{4 m 1}=-C_{m 1}$, and $C_{4 m 2}=-C_{m 2}$. Assume $Z_{m 1}=M_{m 1} s, Z_{m 2}=M_{m 2} s, Z_{s}=M_{s} s$, and let us make the following choices for the controllers:

$$
\begin{aligned}
C_{m 1} & =\frac{K_{p m 1}+K_{v m 1} s}{s}, \quad C_{m 2}=\frac{K_{p m 2}+K_{v m 2} s}{s}, \\
C_{s} & =\frac{K_{p s}+K_{v s} s}{s}
\end{aligned}
$$

The impedance matrix of position-position control dual-user teleoperator can be found from (20), (21), and (22) as

$$
\left[\begin{array}{c}
F_{h 1} \\
F_{h 2} \\
F_{e}
\end{array}\right]=\left[\begin{array}{lll}
Z_{11} & Z_{12} & Z_{13} \\
Z_{21} & Z_{22} & Z_{23} \\
Z_{31} & Z_{32} & Z_{33}
\end{array}\right]\left[\begin{array}{c}
V_{h 1} \\
V_{h 2} \\
V_{e}
\end{array}\right]
$$

In the next subsections, we will consider this position-position shared control architectures and analyze its stability based on both Theorem 1 and Theorem 2. 


\subsection{Stability analysis via passivity criterion (Theorem 1)}

One can see that all the elements of the impedance matrix (24) have only a simple pole on the imaginary axis, thus, Conditions 1 and 2 of Theorem 1 are fulfilled. Analysis of the residues in Condition 2 of Theorem 1 leads to the following conditions

$k_{11}=K_{p m 1} \geq 0, \quad k_{22}=K_{p m 2} \geq 0, \quad k_{33}=K_{p s} \geq 0$

$\frac{k_{11} k_{22}-k_{12} k_{21}}{k_{11}}=\left(1-\alpha+\alpha^{2}\right) K_{p m 1} K_{p m 2} \geq 0$

$\frac{k_{11} k_{33}-k_{13} k_{31}}{k_{11}}-\frac{k_{11} k_{23}-k_{21} k_{13}}{k_{11} k_{22}-k_{12} k_{21}} \frac{k_{11} k_{32}-k_{31} k_{12}}{k_{11}}=0$

The inequality (26) always holds for all $\alpha \in[0,1]$.

Analysis of the impedance matrix (24) according to passivity conditions (2a)-(2d) lead to

$$
\begin{aligned}
& K_{v m 1} \geq 0, \quad K_{v m 2} \geq 0, \quad K_{v s} \geq 0 \\
& 4 K_{v m 1} K_{v m 2}-\left(K_{v m 1}-\alpha K_{v m 1}+\alpha K_{v m 2}\right)^{2} \\
& -\frac{\left(K_{p m 1}-\alpha K_{p m 1}+\alpha K_{p m 2}\right)^{2}}{\omega^{2}} \geq 0
\end{aligned}
$$

It is easy to see that (29) will be fulfilled for all frequencies $\omega$ if the gains of the PD controllers in (23) satisfy

$$
\begin{aligned}
& (1-\alpha) K_{p m 1}=\alpha K_{p m 2} \\
& 4 K_{v m 1} K_{v m 2}-\left(K_{v m 1}-\alpha K_{v m 1}+\alpha K_{v m 2}\right)^{2} \geq 0
\end{aligned}
$$

On the other hand, under (30), Condition (2e) becomes

$$
\begin{aligned}
& -\frac{1}{2 \alpha \omega^{2}}\left(K_{p m 1}-K_{p s}\right)^{2}\left[K_{v m 1}(1-\alpha)^{2}(2-\alpha)\right. \\
& \left.+K_{v m 2} \alpha^{2}\left(1+\alpha^{3}\right)\right]-\frac{1}{\alpha^{2} \omega^{2}}(1-2 \alpha)^{2} K_{v m 1} \\
& -\frac{1}{2 \alpha \omega^{2}}(1-2 \alpha)(1-\alpha)\left(K_{p m 1}^{2}-K_{p s}^{2}\right)\left[\alpha^{2} K_{v m 2}\right. \\
& \left.+(\alpha+2) K_{v m 1}\right]+(1+\alpha)(2-\alpha) K_{v m 1} K_{v m 2} K_{v s} \\
& -\alpha^{2}(2-\alpha) K_{v m 2} K_{v s}\left(K_{v m 2}+K_{v s}\right) \\
& -\left(1-\alpha+\alpha^{2}\right) K_{v m 1} K_{v m 2}\left[(1-\alpha) K_{v m 1}+\alpha K_{v m 2}\right] \\
& -(1-\alpha)^{2}(1+\alpha) K_{v m 1} K_{v s}\left(K_{v m 1}+K_{v s}\right) \geq 0
\end{aligned}
$$

It is easy to see that both (30) and (32) will be fulfilled for all frequencies $\omega$ if the parameters satisfy

$\alpha=\frac{1}{2}, \quad K_{p m 1}=K_{p m 2}=K_{p s}, \quad K_{v m 1}=K_{v m 2}=K_{v s}$

So, a sufficient, frequency-independent, and compact condition for passivity of the above-described position-position dual-user teleoperator is given by (33).

\subsection{Stability analysis via absolute stability criterion (Theorem 2)}

In this case, it can be shown that the symmetrization condition (8) will hold only if $\alpha=\frac{1}{2}$. It is easy to see that the absolute stability conditions (9a)-(9d) become

$$
\begin{aligned}
& K_{v m 1} \geq 0, \quad K_{v m 2} \geq 0, \quad K_{v s} \geq 0 \\
& \frac{7}{8} K_{v m 1} K_{v m 2}+\frac{1}{8 \omega^{2}} K_{p m 1} K_{p m 2}-\frac{Q_{m 1} Q_{m 2}}{8 \omega^{2}} \geq 0
\end{aligned}
$$

where $Q_{m 1}=\sqrt{K_{v m 1}^{2} \omega^{2}+K_{p m 1}^{2}}, Q_{m 2}=\sqrt{K_{v m 2}^{2} \omega^{2}+K_{p m 2}^{2}}$, and $Q_{s}=\sqrt{K_{v s}^{2} \omega^{2}+K_{p s}^{2}}$. Now, under (34), condition (35) will be fulfilled for all frequencies $\omega$ if the gains of the PD controllers $C_{m 1}$
Table 1: The controllers gains of the position-position system used in simulations. (A) Passive and absolutely stable, (B) Absolutely stable but non-passive, (C) Potentially unstable (i.e., not absolutely stable) and non-passive.

\begin{tabular}{l|ll|ll|ll}
\hline & \multicolumn{2}{|c|}{ Master \#1 } & \multicolumn{2}{c|}{ Master \#2 } & \multicolumn{2}{c}{ Slave } \\
\hline (A) & $K_{p m 1}$ & 30 & $K_{p m 2}$ & 30 & $K_{p s}$ & 30 \\
& $K_{v m 1}$ & 5 & $K_{v m 2}$ & 5 & $K_{v s}$ & 5 \\
\hline (B) & $K_{p m 1}$ & 3 & $K_{p m 2}$ & 30 & $K_{p s}$ & 150 \\
& $K_{v m 1}$ & 5 & $K_{v m 2}$ & 50 & $K_{v s}$ & 150 \\
\hline (C) & $K_{p m 1}$ & 3 & $K_{p m 2}$ & 80 & $K_{p s}$ & 15 \\
& $K_{v m 1}$ & 5 & $K_{v m 2}$ & 20 & $K_{v s}$ & 60 \\
\hline
\end{tabular}

and $C_{m 2}$ satisfy

$$
7-4 \sqrt{3} \leq \frac{K_{p m 1}}{K_{v m 1}} \frac{K_{v m 2}}{K_{p m 2}} \leq 7+4 \sqrt{3} .
$$

On the other hand, condition $(9 \mathrm{e})$ will be fulfilled for all frequencies $\omega$ if the gains of the PD controllers $C_{m 1}, C_{m 2}$, and $C_{s}$ satisfy

$$
\frac{K_{p m 1}}{K_{v m 1}}=\frac{K_{p m 2}}{K_{v m 2}}, \quad 5-2 \sqrt{6} \leq \frac{K_{p m 1}}{K_{v m 1}} \frac{K_{v s}}{K_{p s}} \leq 5+2 \sqrt{6}
$$

Clearly, (36) holds if (37) holds. So, a sufficient, frequencyindependent, and compact condition for absolute stability of the above-described position-position dual-user teleoperator is given by (37) where all control gains are nonnegative. Note that the ratios in (37) are merely artifacts of our presentation of the stability conditions meaning that division by zero is avoidable.

\section{Simulations}

In this section, the passivity and absolute stability conditions for the dual-user teleoperator found in the previous section will be verified via simulations. For checking the passivity of the trilateral haptic system, a passivity observer that calculates the dissipated energy in the system has been incorporated into the simulations. The dissipated energy is given by the input-output energy integral

$$
\begin{aligned}
E_{p}(t) & =\int_{0}^{t} F_{h 1}(\tau) V_{h 1}(\tau) \mathrm{d} \tau+\int_{0}^{t} F_{h 2}(\tau) V_{h 2}(\tau) \mathrm{d} \tau \\
& +\int_{0}^{t} F_{e}(\tau) V_{e}(\tau) \mathrm{d} \tau \geq 0
\end{aligned}
$$

The system is passive if the energy integral is non-negative at all time [19].

For checking the absolute stability of the trilateral haptic systems, the ports \#2 and \#3 were connected to passive terminations while the input energy at the port \#1 (i.e., the energy dissipation in the three-port network terminated in ports 2 and 3 ) was measured. The system is absolutely stable if and only if, at all times $t>0$, we have [19]:

$$
E_{S}(t)=\int_{0}^{t} f_{h 1}(\tau) V_{h 1}(\tau) \mathrm{d} \tau \geq 0 .
$$

The position-position dual-user teleoperation system has been simulated in MATLAB/Simulink. There is no time delay in the communication channel between the masters and the slave. Three 1-DOF robots as the two masters and the slave are modeled by masses $M_{m 1}=0.7, M_{m 2}=0.9$, and $M_{s}=0.5$, respectively. In simulations for both passivity and absolute stability, the master \#2 and the slave are connected to LTI terminations with transfer functions $\frac{1}{s+1}$, which are passive as, for $s=j \omega$, we have $\operatorname{Re}\left(\frac{1}{s+1}\right)=\frac{1}{\omega^{2}+1}>0$ when $\omega>0$. In passivity simulations, the master \#1 is connected to another passive LTI termination with transfer functions $\frac{1}{s+1}$, and a sine-wave input $F_{h 1}^{*}$ is applied. In absolute stability simulations, port 1 is open and a sine-wave input $F_{h 1}$ is applied to the master \#1. 

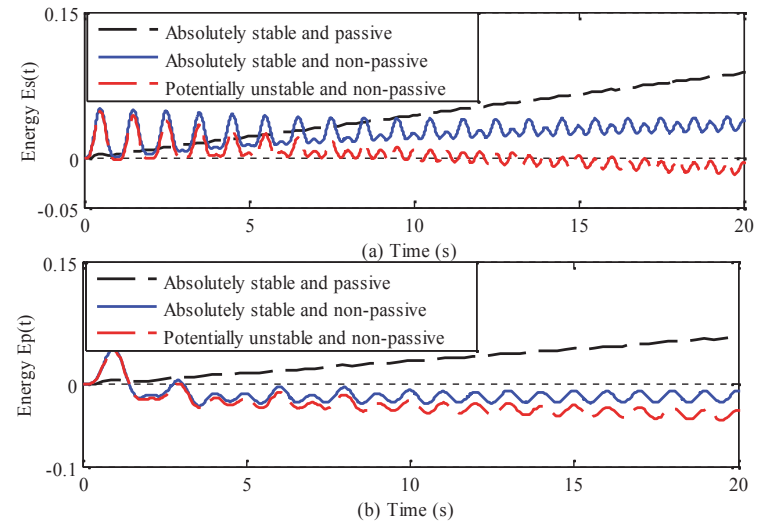

Figure 3: (a) Input energy $E_{s}$ at the master \#1's port used for absolute stability analysis, and (b) passivity observer output $E_{p}$ for used for passivity analysis. Both plots pertain to a position-position dual-user teleoperator. Simulation parameters are listed in Table I: Parameters (A) for the absolutely stable and passive case, parameters (B) for the absolutely stable and non-passive case, and parameters (C) for the potentially unstable and non-passive case.
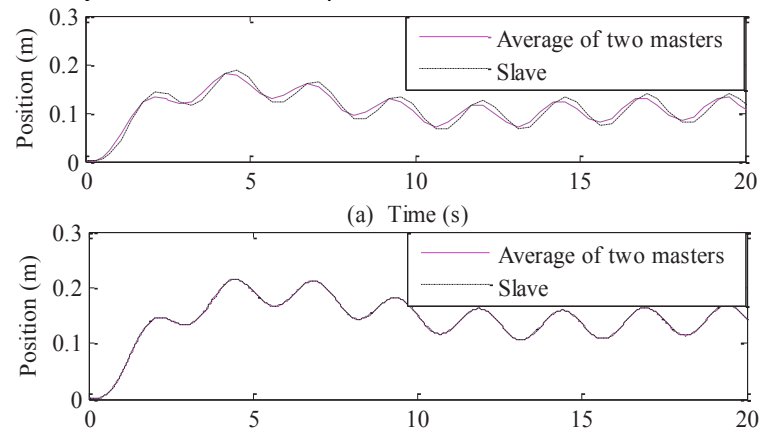

(b) Time (s)

Figure 4: The master robots are shaken intentionally and the slave robot follows the motion of the masters arms. (a) Based on the controllers in Table I (A), (b) Based on the controllers in Table I (B).

According to (33) and (37), the stability of the position-position dual-user teleoperation system should depend on the controllers gains. In the simulations, the controllers gains $K_{p m 1}, K_{v m 1}, K_{p m 2}$, $K_{v m 2}, K_{p s}$, and $K_{v s}$ were chosen according to Table 1 . Also, $\alpha=\frac{1}{2}$.

The input energy (39) profiles $E_{s}$ are plotted in Figure 3(a). As it can be seen, if the controllers gains are selected according to (37), i.e., as listed in Table 1(A) and (B), then the input energy at port 1 is positive at all times, indicating the absolute stability of the trilateral haptic system. However, when the controllers gains violate (37), i.e., as listed in Table $1(\mathrm{C})$, then the input energy $E_{S}$ will become negative at least for a period of time, indicating potential instability of the trilateral haptic system.

The dissipated energy (38) profiles are plotted in Figure 3(b). As it can be seen, if the controllers gains are selected according to (33), i.e., as listed in Table 1(A), then the passivity observer output $E_{p}$ is positive at all times. However, when the controllers gains violate (33), i.e., as listed in Table 1(B) and (C), then the passivity observer output $E_{p}$ is not always positive, indicating the loss of passivity of the haptic teleoperator.

Figure 4 depicts the average positions of the two masters versus the slave position. Figure 4(a) shown the performance for dual-user teleoperation system based on the controllers gains in Table 1(A), and in the first 20 seconds, the integrated squared error of positions is $3.78 \times 10^{-3}$. Figure 4(b) shows the performance based on the controllers gains in Table 1(B), and in the first 20 seconds, the integrated squared error of positions is $3.32 \times 10^{-4}$. Evidently, for designing stabilizing controllers for a trilateral haptic system, we have reached better performance using the absolute stability criterion than using the passivity criterion.

\section{CONCLUSIONS}

In this paper, we showed that the absolute stability criterion is less conservative than the passivity criterion and that the two criteria become the same when a trilateral haptic system is modeled by a symmetric immitance matrix. Both analytically and through simulations involving dual-user haptic teleoperation of one slave robot, the two criteria were compared. It was concluded that the absolute stability criterion is less conservative and allows for better performance compared to the passivity criterion for teleoperators.

\section{REFERENCES}

[1] G. Raisbeck, "A definition of passive linear networks in terms of time and energy," Journal of Applied Physics, vol. 25, no. 12, pp. 15101514, December 1954.

[2] F. Llewellyn, "Some fundamental properties of transmission systems," Proceedings of the IRE, vol. 2, no. 1, pp. 271-283, 1952.

[3] R. F. Kuo and T. H. Chu, "Unconditional stability boundaries of a three-port network," IEEE Trans MTT, vol. 58, pp. 363-371, Dec 2010.

[4] B. Khademian and K. Hashtrudi-Zaad, "Unconditional stability analysis of dual-user teleoperation systems," in 2010 IEEE Haptics Symposium, March 2010, pp. $161-166$.

[5] W. Ku, "Stability of linear active nonreciprocal n-ports," J. Franklin Inst., no. 276, pp. $207-224,1963$.

[6] Y. Wang, F. Sun, H. Liu, and Z. Li, "Passive four-channel multilateral shared control architecture in teleoperation," in 2010 9th IEEE ICCI, July 2010 , pp. $851-858$.

[7] M. Shahbazi, H. A. Talebi, and M. J. Yazdanpanah, "A control architecture for dual user teleoperation with unknown time delays: A sliding mode approach," in 2010 IEEE/ASME International Conference on AIM, July 2010, pp. $1221-1226$.

[8] V. Mendez and M. Tavakoli, "A passivity criterion for n-port multilateral haptic systems," in 2010 49th IEEE Conference on Decision and Control (CDC), December 2010, pp. $274-279$.

[9] H. Anton and C. Rorres, Elementray Linear Algebra: Applications Version. Wiley, 2005, vol. 9.

[10] D. Liberzon, J. Hespanha, and A. Morse, "Stability of switched systems: a lie-algebraic condition," Systems amd Control Letters, vol. 37, pp. 117-122, 1999.

[11] H. K. Khalil, Nonlinear Systems. Prentice Hall, 2002.

[12] S. Haykin, Active Network Theory. Addison-Wesley, 1970.

[13] J. Li, M. Tavakoli, and Q. Huang, "Stability analysis of trilateral haptic collaboration," in IEEE World Haptics Conference 2013, April 2013.

[14] D. Youla, "A note on the stability of linear, nonreciprocal n-port," Proc. IRE, vol. 48, pp. 121-122, 1960.

[15] D. Youla, "A stability characterization of the reciprocal linear passive n-port," Proc. IRE, vol. 47, pp. 1150-1151, 1959.

[16] R. J. Duffin, D. Hazony, and N. Morrison, The Gyration Operator in Network Theory. Scientific Report No. 7, AF 19 (628) 1699, CRST I Sills Bld 5285 Port Royal Road, Springfield, Virginia, 1965.

[17] S. Nudehi, R. Mukherjee, and M. Ghodoussi, "A shared-control approach to haptic interface design for minimally invasive telesurgical training," IEEE Transactions on CST, vol. 13, no. 4, pp. 588-592, July 2005.

[18] M. Tavakoli, A. Aziminejad, R. Patel, and M. Moallem, "High-fidelity bilateral teleoperation systems and the effect of multimodal haptics," IEEE Transactions on SMC, Part B: Cybernetics, vol. 37, no. 6, pp. 1512 -1528, December 2007.

[19] H. J. Marquez, Nonlinear Control Systems Analysis and Design. Wiley, 2003. 